\title{
Simulation Study of Selected Harmonic Eliminated PWM Methods for Cascaded Multilevel Inverters
}

\author{
LI Song \\ Department of Electrical Engineering East China jiaotong University Nanchang, 330013,China \\ email: jxlisong1@163.com
}

Keywords: Selected Harmonic Eliminated; compute online; cased multilevel inverter; interpolation

\begin{abstract}
Calculation switching angles online for Selected Harmonic Eliminated technology was achieved in this paper, using linear interpolation method and Newton-Laphson iteration method respectively. Firstly, change SHE nonlinear equations into algebraic polynomial equations, which is for seven-level inverter, obtain all the solutions using Solve function in Matlab software when the modulation index $\mathrm{m}$ arrange from 0.485 to 1.07 . Then draw a cure for switching angles versus $\mathrm{m}$ and the characteristic of switching angles has been analyzed. Finally, use two different methods, the one that look-up table combining with linear interpolation and the other that the Newton-Laphson iteration method whose initial value decided by equations form a linear fit of switching angles, to compute the angles online for cascaded 7-level inverter controlling by SHEPWM, a comparative analysis has been done. The study of simulation and experiment results certificate the angles worked out by the opposed method are correct. The two methods, mentioned by this paper are valid.
\end{abstract}

\section{Introduction}

Selected Harmonic Eliminated (SHE) Technology in Multilevel Inverters has numerous benefits, such as low switching frequency and low switching loss but a high output quality and efficiency. This method has been drawing more and more attention in Pulse Width Modulation(PWM) control of converters, especially in Multilevel Inverters that applied in high-voltage power occasion. But, the primary problem which limiting SHEPWM to apply to the actual project is solving nonlinear equations. As to this problem, a massive research has been done.

A initial value is very important to iterative algorithm using in solving nonlinear equations, several approaches has been proposed to obtain appropriate ones. Those methods, showing in works [1-3],can reduce the blindness but increase the reliability greatly when choosing a proper initial value. Homotopy algorithm in [4,5] has been applied to solve SHE equations. For its wide convergence domain, this method has a less demanding on the initial value and has been widely used in SHEPWM.I n [6-8],nonlinear transcendental equations under the Fourier domain are converted into piecewise linear equations under Walsh domain, using the relationship between them. This method increase the likelihood of the real-time control of SHEPWM technology by Computer. a lot of work about SHEPWM technology had been done and several key problem was solved. But for the online implementation of the technology, it is rarely mentioned in the literature, what's the main reason is that solving nonlinear transcendental equations online is difficult. Paper [9] mentioned that this online technique could be achieved with a lookup table. However, the problem is that it can not get a series of reasonable solution, which hindered its a further study on it, another approach is mentioned in $[10,11]$. This is done by first converting the transcendental equations that specify the harmonic elimination problem into an equivalent set of polynomial equations. Then, using the mathematical theory of resultants, all solutions to this equivalent problem can be found. No initial value is needed in the method, however, there's a large computation when solving polynomial equations.

As mentioned above, firstly convert the transcendental equations into an equivalent set of polynomial equations and using symbolic computation to solve polynomial equations, then we can obtain all the possible solutions, which open the door to implement SHEPWM online. After referring to the formers' achievement in research, we get all solutions of solve polynomial 
equations in this work by using the function Solve that offered special for polynomial equations in mathematic software Matlab. This approach turns out to be simple and efficiency, additionally, it performs good as evaluated by the total harmonic distortion (THD) of inverter outputs and high magnitude of the output fundamental frequency component. In this paper, all the set of solutions for a Cascaded seven-level inverter, which not generating the 5th and 7th harmonic, were obtained by the proposed technique. Then, draw a cure for switching angles versus modulation index $\mathrm{m}$, choose a set of reasonable switching angles, and achieve calculating switching angles online for SHEPWM using linear interpolation method and Newton-Laphson iteration method respectively. Finally, a comparative analysis between the two online methods has been done, which have practical significance for SHEPWM controlling online.

\section{Cascaded Multilevel Inverters and SHEPWM}

Cascaded H-Bridge Multilevel Inverter uses several single-phase full-bridge with Separate DC (SDCs) sources. This topology synthesizes a desired voltage waveform from independent sources of DC voltages, which may be obtained from batteries, fuel cells, or solar cells. The AC voltage terminals of each full-bridge unit are connected in series. Each units can generate three different output voltages $+\mathrm{E}, 0$,-E. The synthesized total AC voltage output waveform is the sum of the individual inverter voltage outputs. In this Multilevel Inverter topology, the number of output phase voltage levels is defined by $\mathrm{N}=2 \mathrm{H}+1$, where $\mathrm{H}$ is the number of DC sources and $\mathrm{N}$ is the number of inverter levels. An example of single phase topology for a seven-level cascaded inverter with three full-bridges units is shown in the Fig.1 and stepped wave form in Fig.2.

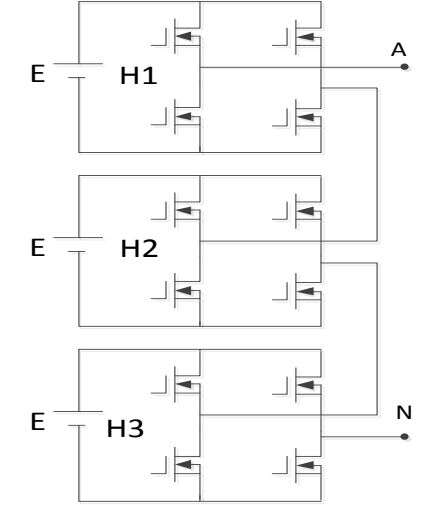

Fig. 1 The topology of H-Bridge seven-level inverter

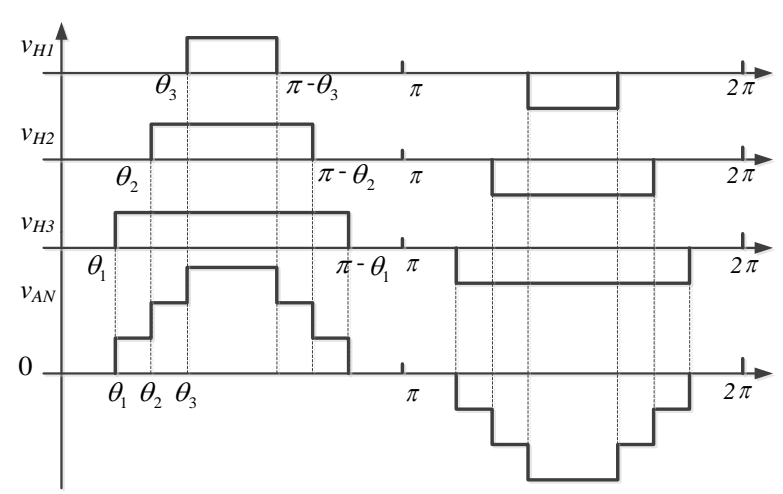

Fig.2 Staircase wave synthesized model for Cascaded 7-level inverters

The Fourier series of staircase wave of the multilevel inverter is written as follows:

$$
u(\omega t)=a_{0}+\sum_{n=1}^{\infty}\left(a_{n} \cos (n \omega t)+b_{n} \sin (n \omega t)\right)
$$

The DC part $\mathrm{a} 0=0$, as the area of the positive and negative half cycle of the waveform are equal. Cosine component and even order sine component are zero, it says an=0and the fundamental wave and each odd order sine harmonic component are as follows:

$$
\left\{\begin{array}{l}
b_{n}=0, n \rightarrow \text { even } \\
b_{n}=\frac{4 E}{n \pi} \sum_{k=1,3,5,5}^{N} p_{k} \cos \left(n \theta_{k}\right), n \rightarrow \text { odd }
\end{array}\right.
$$

The switching angles, denoted by $\theta \mathrm{k}$ (in radians),namely $0<\theta 1<\theta 2<\cdot \bullet \cdot<\theta \mathrm{N}$; E represent the value of DC voltage. pk is marked +1 when it meet a rises edge and -1 when it falls.

Substituting the values of $\mathrm{a} 0$, an and bn the instantaneous output voltage equation can be obtained.

$$
u(w t)=\frac{4 E}{n \pi} \sum_{k=1,3,5, \ldots}^{N} p_{k} \cos \left(n \theta_{k}\right) \sin (n w t)
$$


The modulation index is defined by $m=\frac{\pi \cdot b_{1}}{H \cdot 4 E}$, which $H$ is the number of DC sources. As stepped waveform shown in Fig.2,m for cascaded H-bridge seven-level inverter range from 0.485 to 1.07[12].

According to equation(2) and (3),the SHE model for seven-level inverter can been simplified as the following equation(3).

$$
\left\{\begin{array}{l}
\cos \left(\theta_{1}\right)+\cos \left(\theta_{2}\right)+\cos \left(\theta_{3}\right)=\frac{3 \pi}{4} \cdot m \\
\cos \left(5 \theta_{1}\right)+\cos \left(5 \theta_{2}\right)+\cos \left(5 \theta_{3}\right)=0 \\
\cos \left(7 \theta_{1}\right)+\cos \left(7 \theta_{2}\right)+\cos \left(7 \theta_{3}\right)=0
\end{array}\right.
$$

In paper[13], a formulation was derived, which make it easier to cosine function of moltiple angles replaced as cosine function. the general formula as follow:

When $\mathrm{n}$ is odd,

$$
\cos (n \theta)=\sum_{h=0}^{n-1 / 2}\left(C_{n-h}^{h}-C_{n-1-h}^{h-1}\right)(-1)^{h} 2^{n-1-2 h} \cos ^{n-2 h} \theta
$$

$\mathrm{n}$ is even,

$$
\cos (n \theta)=\sum_{h=0}^{n / 2}\left(C_{n-h}^{h}-C_{n-1-h}^{h-1}\right)(-1)^{h} 2^{n-1-2 h} \cos ^{n-2 h} \theta
$$

Cosine function of 5 times angle and 7 times angle in equation(3) can be respectively resolved into equation(6) and equation(7):

$$
\begin{aligned}
& \cos (5 \theta)=5 \cos (\theta)-20 \cos ^{3}(\theta)+16 \cos ^{5}(\theta) \\
& \cos (7 \theta)=-7 \cos (\theta)+56 \cos ^{3}(\theta)-112 \cos ^{5}(\theta)+64 \cos ^{7}(\theta)
\end{aligned}
$$

Let $: \cos (\theta 1)=x 1, \cos (\theta 2)=x 2, \cos (\theta 3)=x 3$,

Substituting (6)and(7) into equations(3), the new SHE model come out to be equations (8), Nated that $1>x 1>x 2>x 3>0$.

$$
\left\{\begin{array}{l}
x_{1}+x_{2}+x_{3}=\frac{3 \pi}{4} \cdot m \\
\sum_{i=1}^{3} 5 x_{i}-20 x_{i}^{3}+16 x_{i}^{5}=0 \\
\sum_{i=1}^{3}-7 x_{i}+56 x_{i}^{3}-112 x_{i}^{5}+64 x_{i}^{7}=0
\end{array}\right.
$$

\section{Solve Polynomial Equations}

There're three basic types of methods to solve polynomial equations, including numerical solution, symbols solution and the mixed one by the forenamed two[14].In order to obtain all the possible real solution of SHE model, symbols solution was utilized to calculate the equations. Traditional symbols arithmetic, to solve Algebraic equations have the same disadvantages, that large calculation, low operational speed and hard to programmed. Here ,function Solve, that is special for getting the analytic solution by mathematic software Matlab, has been used to solve the SHE model. It simplifies the whole calculation procedure. What's more, this proposed approach don't need a starting value, compared to the iterative algorithm. SHE model for cascaded seven-level inverter was Taken as an example to show how the program works using the mentioned method. Procedures are as follows:

1) Substitute modulation index $m$ into (8);

2 ) Exploit Function Solve to get the roots of polynomial equations and named as $\mathrm{x} 1, \mathrm{x} 2, \mathrm{x} 3$, respectively;

3) Operate $x 1 、 x 2 、 x 3$ by arccosine, then we get the solutions of trigonometric equations, expressed as $\left[\begin{array}{lll}\theta 1 & \theta 2 & \theta 3\end{array}\right]=[\arccos (\mathrm{x} 1) \quad \arccos (\mathrm{x} 2) \quad \arccos (\mathrm{x} 3)]$, where $\theta 1 、 \theta 2$ and $\theta 3$ are the angles 
for seven-level inverter.

Follow Procedures above, we can find out all possible solutions for CHB seven-level inverter modulated by SHEPWM and the modulation index $m \in[0.485$ 1.07].Results for the Seven-Level Inverter are summarized in Fig. 3 which shows the switching angles $\theta 1 、 \theta 2$ and $\theta 3$ versus m. The parameter $\mathrm{m}$ was incremented in steps of 0.01 ,expressing as $\Delta \mathrm{m}=0.01$. In Fig.3 the symbols' + ' '*'、 'o' are represent for orbits of $\theta 1, \theta 2, \theta 3$,respectively and the horizontal axis represents the modulation index $\mathrm{m}$, while the vertical axis represents the angles. As Fig. 3 shows, when $\mathrm{m}$ ranges from 0.637 to 0.786 ,there're two different sets of solutions. In order to analyze the characteristics of results, all the switching angles were individually simulated and THD of line-line voltage for each set of roots was recorded. The relationship between THD of line-line voltage and modulation index $\mathrm{m}$ is shown in Fig.4.As we can see in the graph, THD of line-line voltage is under 20 percents, in the whole range of $\mathrm{m}$. And, in Most range of $\mathrm{m}$, the first group of solution perform better than the second one, evaluated by THD.

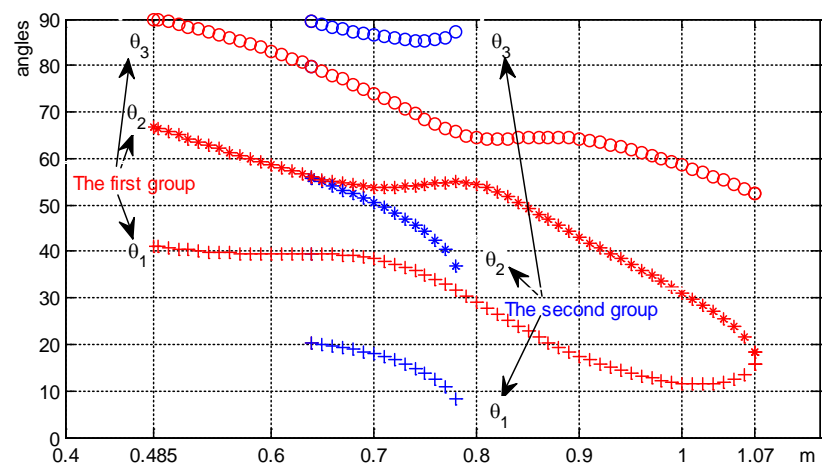

Fig.3 Switching angles track vs $\mathrm{m}$ for SHEPWM Control Technology of 7-level inverters

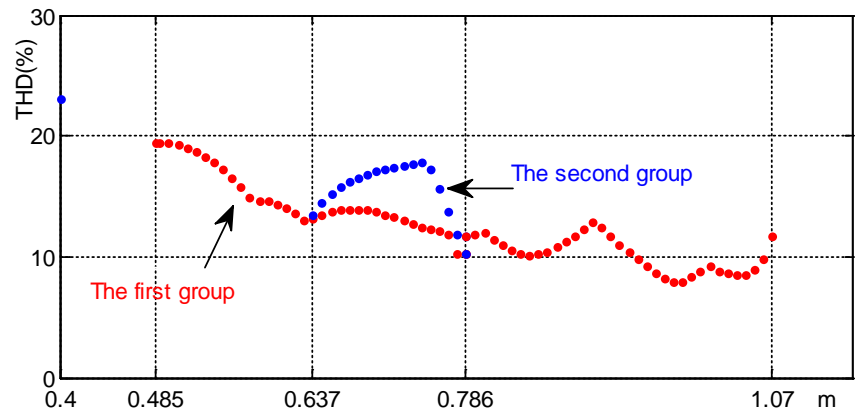

Fig.4 The curve of THD for line-line output voltage vs the modulation index $\mathrm{m}$

\section{Simulation Investigation}

In order to study waveforms of phase voltage VAN and line-to-line voltage VAB and it's THD, simulations for CHB NPC three-level inverter are carried out in Matlab/Simulink. Three angle for a CHB seven-level inverter controlled by SHEPWM had been work out ,that is $\theta 1=21.79956^{\circ}, \theta 2=48.3429^{\circ}$ and $\theta 3=64.63573^{\circ}$,exploiting the method opposed in this section, when $\mathrm{m}$ is 0.863.Next,Simulation experiment using Matlab/Simulink had been done to demonstrate the validity of the approach. Simulation parameters are as follow: the value of DC voltage is $50 \mathrm{~V}$,the fundamental frequency is $50 \mathrm{~Hz}$,with a pure resistance load. The result of simulation experiment show in Fig.5 and Fig.6. 

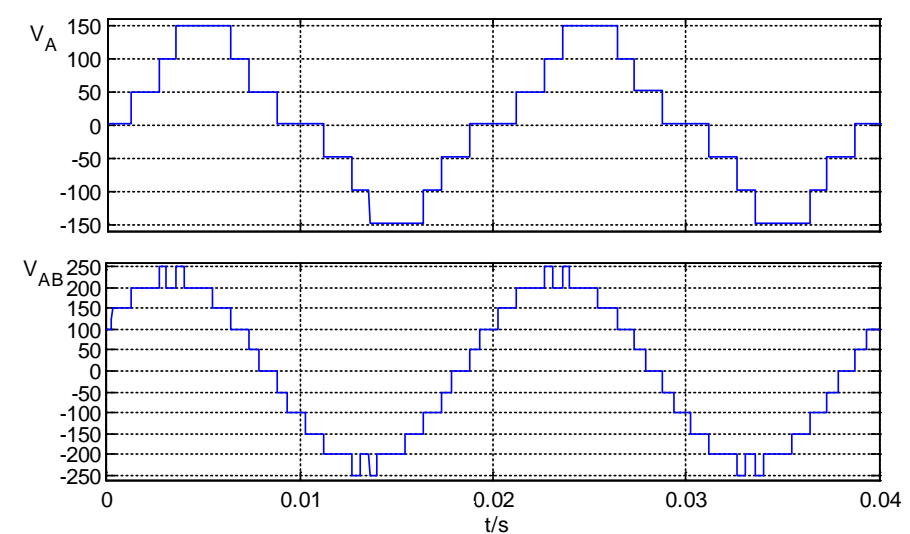

Fig.5 The waveform for phase and line-line output voltage to the calculate result using linear interpolation method

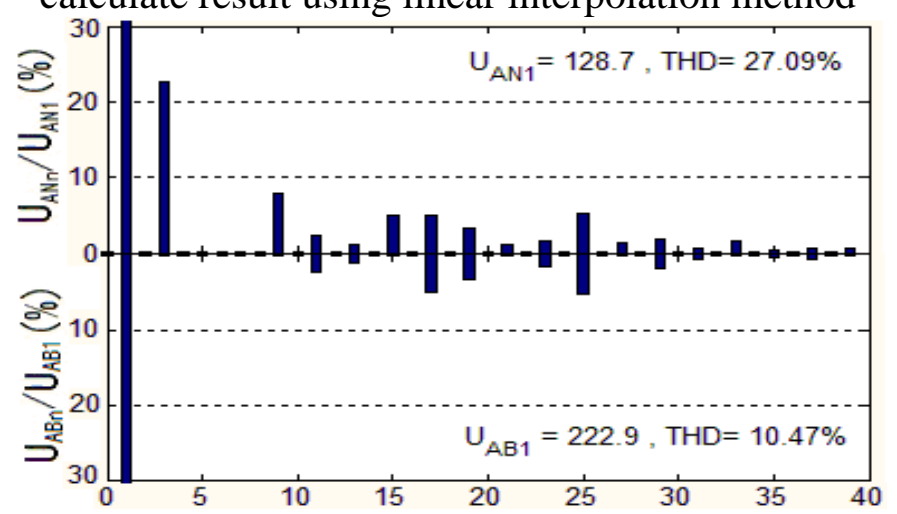

Fig.6 The spectrum of output voltage to the calculate result using linear interpolation method

We can see, 5th and 7th are almost eliminated, shown in the spectrum in Fig.6 All above proved that the approach mention in this section is feasible and effective. Noted that two process cannot forget, one is exploiting function Solve to get the solutions from the converted polynomial equations, the other is setting up the data chart.

\section{Conclusions}

A full solution to the problem of eliminating the fifth and seventh harmonics in a seven level multilevel inverter has been given. Specifically, function Solve has used to solve the converted polynomial equations. Further, achieve calculating switching angles online for Selected Harmonic Eliminated technology.It is feasible to use function Solve in Matlab solve the converted polynomial equations and no initial angles will be needed and programming is relatively simple (in contrast to numerical techniques such as Newton-Raphson or Homotopy algorithm), furthermore, a full solution can be obtained.

\section{Acknowledgement}

This work is supported by the visiting scholar special fund projects of young teachers development plan in Jiangxi Province Universities .

\section{References}

[1] Fei Wanmin, Ruan xinbo, Zhang Yanli, et al. Research on the initial values of SHEPWM method for multi-level voltage inverters[J]. Proceeding of the CESS, 2007, 27(13): 87-92(in Chinese).

[2] Fei Wanmin,Zhang Yanli,Dou Xiaoli.Selective Harmonic Elimination PWM Method for Five-Level Inverters[J].Trans of China Electronics Society.2009, 24(2): 85-99.

[3] WU ChunhuiJIANG Qiong ZHANG Chunpeng. An optimization method for threelevel 
Selective Harmonic Eliminated Pulse Width Modulation SHEPWM [C]//2005 International Conference on Electrical Machines and Systems. Nanjing China IEEE 200513461350

[4] Xie Yunxiang, Zhou Lian, Peng Hong. Homotopy algorithm research of the inverter harmonic elimination PWM model[J]. Proceeding of the CSEE, 2000, 20(10): 23-26.

[5] Zhu Meijie, Zhao Ping, Peng Hong, et al. Fast Homopoty algorithm of the inverter harmonic elimination PWM model[J]. Journal of south China University of technology(Natural Science Edition), 2004, 32(2): 28-21.

[6] Swift F, Kamberis A. A new Walsh domain technique of harmonicelimination and voltage control in pulse-width modulated Inverters[J]. IEEE Trans on Power Electronics, 1993, 8(2): 170-185.

[7] Liang $\mathrm{T} \mathrm{J}$, Hoft $\mathrm{R} \mathrm{G}$. Walsh function method of Harmonic Elimination[C].IEEE APEC'93.CA: San Diego, 1993: 847-853.

[8] Zheng Chunfang, Zhang Bo, Qiu Dongyuan. Selective Harmonic Elimination Technique Based on Walsh Transform for Multilevel Inverters[J].Trans of

China Electronics Society, 2006, 21(7): 121-126(in Chinese).

[9] Song Pinggan, Guan Eryong. Selective harmonic elimination for all Modulation indices of multilevel cascaded converter[J]. Trans of China Electronics Society, 2006, 21(9): 81-87.

[10] Chiasson J N, Tolbert L M, McKenzie K, et al. A Complete Solution to the Harmonic Elimination Problem.IEEE Trans. Power Electronics 2003,19(2): 596-603.

[11] Chiasson J N, Tolbert L M, McKenzie K J, et al. A unified approach to solving the harmonic elimination equations in multilevel Converters[J] . IEEE Trans. Power Electronics, 2004, 19(2): 478-490.

[12]YE Manyuan, LI Bing, LI Song.Study of Multilevel inverter Multiband SHE-PWM Control Technology Based on Walsh Function[J].Power system technology.2013.37(10):2945-2952 .

[13] Yuanhong Lu. Sine and Cosine of the Times Angle Formula and Its Applications.College Mathematic[J].2013, 29(3): 23-26. (In Chinese)

[14] Zheng Chun-fang,Zhang Bo. Application of Wu Method to Harmonic elimination Techniques [J].Proceeding of the CSEE, 2005, 25(15): 40-45 